The University of Southern Mississippi

The Aquila Digital Community

Faculty Publications

8-1-2019

\title{
Effects of a Brief Mindfulness Induction On Death-Related Anxiety
}

David M. Schultz

University of Southern Mississippi, david.schultz@eagles.usm.edu

Randolph C. Arnau

University of Southern Mississippi, Randolph.Arnau@usm.edu

Follow this and additional works at: https://aquila.usm.edu/fac_pubs

Part of the Psychology Commons

\section{Recommended Citation}

Schultz, D. M., Arnau, R. C. (2019). Effects of a Brief Mindfulness Induction On Death-Related Anxiety. OMEGA: Journal of Death and Dying, 79(3), 313-335.

Available at: https://aquila.usm.edu/fac_pubs/17104

This Article is brought to you for free and open access by The Aquila Digital Community. It has been accepted for inclusion in Faculty Publications by an authorized administrator of The Aquila Digital Community. For more information, please contact Joshua.Cromwell@usm.edu. 


\title{
Effects of a Brief Mindfulness Induction on Death-Related Anxiety
}

OMEGA-Journal of Death and

2019, Vol. 79(3) 313-335

(C) The Author(s) 2017

Article reuse guidelines: sagepub.com/journals-permissions DOI: I0.1 I77/00302228I772III5 journals.sagepub.com/home/ome

\section{David M. Schultz' and Randolph C. Arnau'}

\begin{abstract}
This study examined effects of a mindfulness induction on proximal and distal defense responses to mortality salience and negative affect. Three experimental conditions were included: mindfulness, mind-wandering, and worrying. Participants in the mindfulness condition underwent a mindfulness induction at the experiment's outset, while participants in the other two conditions underwent a mind-wandering or worry induction. Inductions involved following guided audio instructions presented via headphones. All conditions $(N=77)$ underwent a mortality salience induction after experimental manipulation, involving a written exercise pertaining to one's death. Results indicated fewer proximal responses in the mindfulness and mindwandering groups, compared with the worrying group, but no differences in distal responses. Negative affect was lower in the mindfulness group than in the worrying group following mortality salience. Results suggest that mindfulness exercises effectively buffer against negative affect and some responses to mortality salience, although these effects are not different from those of mind-wandering.
\end{abstract}

\section{Keywords}

mindfulness, terror management theory, anxiety

\footnotetext{
'The University of Southern Mississippi, Hattiesburg, MS, USA
}

\section{Corresponding Author:}

David Schultz, Department of Psychology, The University of Southern Misssippi, I 8 College Drive, \#5025, Hattiesburg, MS 39406, USA.

Email: david.schultz@eagles.usm.edu 


\section{Terror Management Theory (TMT)}

TMT postulates that existential anxiety resulting from knowledge of one's inevitable death is unique to humans (Greenberg, Pyszczynski, \& Solomon, 1986). More importantly, TMT posits that the behavioral effects of the conscious awareness of one's mortality, or mortality salience, occur across multiple domains, including psychopathology, legal decision-making, sexuality, religious behavior (Kesebir, 2014, p.611), and romantic attachment style (Coolsen \& Nelson, 2002). Cognitive and behavioral reactions to mortality salience can vary widely, with many individuals reporting extreme anxiety and distress when confronted with the thought of their own death (Kesebir, 2014).

Although the existence of emotional and behavioral responses to mortality salience is well-documented (Kesebir, 2014; Niemiec et al., 2010, Sliter, Sinclair, Yuan, \& Mohr, 2014), relatively little research has investigated ways to intervene against the negative effects of mortality salience. A series of nine studies by Gailliot, Schmeichel, and Baumeister (2006) established that emotional selfregulation moderates the degree to which individuals experience death-related anxiety in response to a mortality salience induction. It therefore stands to reason that mindfulness may be an effective tool in attenuating defensiveness toward thoughts of mortality because of its inherent means of increasing psychological flexibility and emotional self-regulation.

To date, only one series of published studies directly examined mindfulness and mortality salience (Kesebir, 2014). This series of studies (Niemiec et al., 2010; Sliter et al., 2014) examined the topic from the perspective of trait mindfulness, but no study has yet attempted an experimental intervention to buffer against responses to mortality salience. Thus, this was the purpose of the present study. Specifically, the present study examined the effects that a mindfulness induction (compared with a mind-wandering and worry induction conditions) had on proximal and distal defense responses and negative affect, when administered just prior to a mortality salience induction.

Emotional responses to mortality salience may be especially distressing to vulnerable populations who may consistently have death-related thoughts-for example, suicidal or depressed individuals, individuals with chronic or terminal illness, or those nearing the end of the natural life span. As such, the psychological literature would benefit from experimental research specifically investigating means of buffering against the negative aspects of mortality salience as a means of decreasing negative emotionality in vulnerable populations and improving overall quality of life.

In TMT literature, a sense of personal value that defends against deathrelated anxiety is referred to as self-esteem. Self-esteem comprises possessing a firmly held belief in two factors: First, that one possesses a worldview that is superior to all opposing worldviews or is at least valid and internally consistent to the individual; and second, that one meets the standards of behavior espoused 
by that worldview (Florian \& Mikulincer, 1997). For most individuals, this worldview is provided by the culture with which the individual identifies.

Of particular interest to researchers are what are referred to as proximal and distal defense mechanisms exhibited in subjects who are prompted to be consciously aware of their own mortality. Proximal defense mechanisms typically refer to conscious attempts to suppress or ignore mortality-related thoughts, whereas distal defense mechanisms are conceptualized as alterations in behavior which are relatively indirect or unconscious in nature. Proximal defense mechanisms are relatively easy to define: In general, any behavior which consciously attempts to escape thoughts of death and the affective discomfort brought by these thoughts may be considered a proximal defense mechanism. Conversely, the term "distal defense mechanism" refers to a more abstract category of behaviors undertaken in an effort to reassure the individual that their worldview, ideals, and surrounding environment are consistent with their approach to behavior. The tendency for mortality salient individuals to hand out harsher punishments to hypothetical criminals, for example, is considered a distal defense mechanism because this behavior theoretically represents an unconscious or symbolic attempt by the individual to purge their environment of forces which may pose a threat to their safety, worldview, or society. By doing so, the individual maintains a sense of certainty and security which would otherwise be threatened by mortality salience generated by the threat of criminal activity. Proximal and distal defense mechanisms are often observed as a proxy means of measuring death anxiety in laboratory settings.

It has been established that increased mortality salience can arouse distress, anxiety, and sadness (Burke, Martens, \& Faucher, 2010; Florian \& Mikulincer, 1997; Greenberg, Simon, Pyszczynski, Solomon, \& Chatel, 1992; Kesebir, 2014). Psychological defense responses to these emotions have been well-documented in the literature. A meta-analysis by Burke et al. (2010) found that, on average, across 277 experiments, mortality salience yielded moderate effects $(r=.35)$ on various indices of defensive responses, such as increased aggression toward others who violate their worldview, more negative responses to those who violate their moral beliefs (Florian \& Mikulincer, 1997), and a higher likelihood of bias and favoritism toward those who support their worldview compared with those who have not undergone mortality salience induction (Greenberg et al., 1992; Niemiec et al., 2010). All these reactions to mortality salience, from the perspective of TMT, are considered to function as defenses against the uncomfortable emotions elicited by mortality salience.

Proximal and distal defense responses can be measured in a variety of ways. Proximal defense mechanisms typically refer to active suppression of death or mortality-related thoughts. Previous research has examined proximal defense responses in the contexts of suppression of death thoughts, denial of vulnerability to terminal disease or premature death (Niemiec et al., 2010), as well as positive cognitive and behavioral changes such as increased intention to exercise 
(Arndt, Schimel, \& Goldenberg, 2003) and use sunscreen (Routledge, Arndt, \& Goldenberg, 2004). Conversely, distal defense mechanisms are conceptualized as more unconscious or symbolic in nature than proximal defense responses. Distal defense responses are more closely related to threats to self-esteem or worldview than to explicit death-related thoughts.

\section{Mindfulness and Acceptance}

Scientific interest in mindfulness has drastically expanded in the last few decades (Brown, Ryan, \& Creswell, 2007), particularly in clinical psychology and related fields. Mindfulness-based interventions have shown efficacy in a vast number of contexts, including stress reduction (Grossman, Niemann, Schmidt, \& Walach, 2004) and treatment of depression, anxiety, sexual disorders, eating disorders, drug dependence, attention deficit hyperactivity disorder, and chronic pain (Chambers, Gullone, \& Allen, 2009). A three-point definition offered by KabatZinn (1994, p. 4) defines mindfulness as "paying attention in a particular way: on purpose in the present moment, and nonjudgmentally." The three components of mindfulness postulated in Kabat-Zinn's definition - purposefulness, focus on the present moment, and a nonjudgmental attitude - appear as common threads throughout most definitions of mindfulness.

Mindful individuals are able to view their own thoughts, pleasant or unpleasant, as simply thoughts and are thus able to emotionally respond to them in a more objective and less threatened manner (Brown et al., 2007). In other words, mindfulness allows the individual to process difficult thoughts and feelings in a more objective and regulated manner. By viewing difficult feelings through the lens of mindfulness, individuals are better equipped to analyze difficult thoughts and feelings without the emotional charge that accompanies fear or anxiety.

Individuals who are practiced in mindful thinking tend to possess a greater control over the focus of their attention, allowing them to focus on particular aspects of reality or "zoom out" to view the broader context in which phenomena exist (Brown et al., 2007). This skill, when practiced, leads to increased psychological flexibility, which is associated with the ability to adapt to varying situations, adapt cognitions to fit social contexts or needs, view one's own behaviors objectively, and compare one's own behaviors to one's values (Kashdan \& Rottenberg, 2010).

Some research suggests that the state of mindfulness can be briefly heightened in laboratory settings. Arch and Craske (2006) successfully heightened mindfulness in undergraduate subjects who underwent a 10-minute mindfulness induction compared with subjects who did not. Individuals who underwent the induction went on to show heightened emotional regulation and distress tolerance when exposed to extremely unpleasant visual stimuli later in 
the experiment. Although mindfulness also improves over time with practice, this study indicates that a mindful state can be briefly induced in the laboratory even with individuals that are new to mindfulness practice.

If mindfulness is associated with greater psychological flexibility, emotional regulation, and distress tolerance, it stands to reason that a mindful and accepting attitude toward death-related cognitions may assuage anxiety or other negative affect associated with mortality salience. By altering one's cognitions about death through mindfulness, one may experience lessened distress when confronted with thoughts of death.

As a consequence of this lessened negative affect when presented with mortality salience, one may reasonably predict that that such would in turn be associated with decreased defensive responses to mortality salience. Thus, in the present study, it was predicted that mortality salient individuals in a mindful state would exhibit fewer proximal and distal defensive responses toward thoughts of death than individuals who were not in a mindful state.

\section{Method}

\section{Participants}

To be eligible for participation in the study, participants were required to be 18 years or older, may not have been treated or used psychotropic medication for mental disorders in the last 2 years, and must had have no previous experience in mindfulness or meditation techniques. These eligibility criteria were adapted from Arch and Craske (2006). Potentially eligible students were screened through online survey software and, if eligible, scheduled a time to complete the study in the laboratory setting. A convenience sample of 77 participants was obtained for the experiment. Participants were college students who volunteered to participate in exchange for either required or extra credit for a general psychology course and selected other psychology courses. A cafeteria model is used, in which psychology students log onto an online study scheduling system and choose from a variety of online and laboratory-based psychology studies. Required or extra credit is awarded in accordance with the amount of time and effort involved with a study.

After screening for eligibility, a total of 77 participants completed the experiment. Participants were undergraduate students at The University of Southern Mississippi, recruited through an online subject pool, who received course credit for participation.

The final sample was $80.5 \%$ female and $40.3 \%$ White, $54.5 \%$ Black/AfricanAmerican, $1.3 \%$ Asian/Asian-American, and 3.9\% multiracial. Approximately $62.4 \%$ of the participants were in their first or second year of college. Participant ages ranged from 18 to 46 , with a mean age of $20.7(S D=4.80)$. 


\section{Materials}

Word fragment task. To operationalize proximal defense mechanisms, participants completed a set of 25 word fragments (Arndt, Greenberg, Pyszczynski, \& Solomon, 1997; Greenberg, Pyszczynski, Solomon, Simon, \& Breus, 1994) containing a blank space into which letters could be written. Eight of these word fragments could be completed with either a neutral or death-related word (e.g., KI_ED can be completed with the neutral words [KISSED, KICKED] or with a death-related word [KILLED]). The fragments which could not be filled with death-related words served as filler items to prevent participants from guessing the purpose of the fragment task. This task, adapted from methods used by Niemiec et al. (2010), measured death-thought accessibility and was treated as a proxy for proximal defense responses. Proximal defense responses were scored by counting how many word fragments were completed with a death-related word, with a higher number indicative of a more defensive response. The number of death-related words written by each participant was summed, with their final score on the measure equaling the number of death-related words written. These scores were then compared across conditions during statistical analysis.

Multidimensional Social Transgression Scale (MSTS). Distal defense mechanisms were operationalized using an adaptation of the MSTS developed by Florian and Mikulincer (1997). The scale consists of 20 short vignettes written in the style of a newspaper report. Each vignette describes a major calamity befalling an undeserving victim due to the moral or social transgression of another. Each vignette describes either intrapersonal or interpersonal consequences for the victim. Interpersonal consequences involve extreme damage to one's social standing, family, or friends as a result of the offender's transgression (e.g., a man's social reputation is ruined after falsely testing positive for HIV under the care of an incompetent doctor). Conversely, intrapersonal consequences involve direct damage to the victim, typically in the form of physical or mental harm (e.g., a doctor mixes up the records of two patients and amputates the leg of the wrong one). The original MSTS was written for use in Israel, with many of the original vignettes referring specifically to Israeli culture. For example, one vignette refers to a "kibbutz" with the implicit assumption that the participant will know what the word means. For the present study, all culturally specific vignettes were removed, reducing the number of vignettes to 10. This also mimicked the MSTS as it was used by Niemiec et al. (2010).

After reading a given vignette, the participant rated the severity of the transgression as well as the severity of punishment appropriate for the perpetrator, both on a 7-point scale. Distal defense response was scored by summing both "severity of transgression" and "severity of punishment" scores for all 10 vignettes to create a single, composite score. 
MSTS subscale scores were computed for each of the following four domains: evaluations of (a) severity of personal offenses, (b) severity of punishment for personal offenses, (c) severity of interpersonal offenses, and (d) severity of punishment for interpersonal offenses. Final scores for each participant were derived by reverse coding responses as necessary and then summing their numeric ratings for each vignette as a composite score. Composite scores were compared across conditions during statistical analysis.

Positive Affect Negative Affect Schedule (PANAS). The PANAS (Watson, Clark, \& Tellegen, 1988) was administered at multiple time points to measure state negative affect. With a Cronbach's $\alpha$ coefficient of .84 to .87 and test-retest correlations of .39 to .71 for the Negative Affect subscale (Watson et al., 1988), the PANAS demonstrates strong validity and reliability with regard to general positive and negative affect. Different instruction sets can be used to assess either state or trait affectivity. The instruction set which asks participants to rate how they feel "right now" was used for the current study, which directed test takers to rate emotions as they are experiencing them at that very moment. Scores are derived by reverse coding items as necessary, then summing participants' ratings of all items to arrive at a composite score which was compared across groups during statistical analysis.

Philadelphia Mindfulness Scale (PHLMS). Trait mindfulness was assessed using the PHLMS (Cardaciotto, Herbert, Forman, Moitra, \& Farrow, 2008). The PHLMS is a bidimensional measure developed to assess two key components of mindfulness via two independent subscales: Present-Moment Awareness and Acceptance (Cardaciotto et al., 2008). The scale consists of two 10-item subscales measuring awareness and acceptance, respectively. Each item is rated on a 5-point Likert-type scale $(1=$ never, $5=$ very often $)$ according to the frequency with which they experienced the described item over the past week (Cardaciotto et al., 2008). Both the Awareness subscale and Acceptance subscale demonstrated good internal consistency in a sample of 204 nonclinical undergraduates, with Cronbach's $\alpha$ coefficient of .81 and .85, respectively. The scale has demonstrated convergent, discriminant, concurrent, and predictive validity across both clinical and normal undergraduate populations (Quaglia, Brown, Lindsay, Creswell, \& Goodman, 2015). Scores were derived by reverse coding items as necessary and then summing the numeric ratings of all items on the scale to arrive at a composite score for each participant. Composite scores were compared across groups during statistical analysis.

Kentucky Inventory of Mindfulness Scales (KIMS). A second measure of trait mindfulness was in addition to the PHLMS. The KIMS (Baer, Smith, \& Allen, 2004) is a 39-item self-report measure in which each item is rated on a 5-point Likerttype scale $(1=$ never or very rarely true, 5=almost always or always true) 
according to the extent to which the participant endorses a given trait. The scale has demonstrated high content validity, as well as adequate to good internal consistency, with $\alpha$ coefficients ranging from .76 to .91 across four factors, measuring observation, describing, acting with awareness, and acting without judgment. As on the PHLMS, scores were derived by reverse coding items as necessary and then summing the numeric ratings of all items on the scale to arrive at a composite score for each participant, which were compared across groups during statistical analysis.

Cognitive and Affective Mindfulness Scale-Revised (CAMS-R). A third measure of trait mindfulness was used. The CAMS-R (Feldman, Hayes, Kumar, Greeson, \& Laurenceau, 2007) is a 12-item self-report measure in which each item is rated on a 4 -point Likert-type scale $(1=$ rarely/not at all, $4=$ almost always $)$ according to how often a given item applies to the respondent. The measure has demonstrated an acceptable Cronbach's $\alpha$ of .74 to .77 and shows evidence of convergent and discriminant validity. As on the PHLMS and KIMS, scores were derived by reverse coding items as necessary, then summing participants' numeric ratings of each item to arrive at a composite score which was compared across groups during statistical analysis.

\section{Procedure}

All data were collected between September and December of 2015. The experiment was conducted in a laboratory at The University of Southern Mississippi. Participants sat at a desk with a laptop computer which was used to complete questionnaires and all experimental procedures, including auditory presentation of instructions constituting the experimental manipulations. The experimenter left the room during the experimental manipulations. Completion of all questionnaires, including those administered before and after the experimental manipulations, took approximately 30 to 40 minutes. Audio instructions presented during each experimental manipulation lasted approximately 9 minutes. A mortality salience induction administered to all participants lasted approximately 5 minutes. In total, the full duration of the experiment typically took between 45 and 60 minutes.

After providing informed consent, participants completed the PANAS in order to establish an affect baseline, along with the PHLMS, KIMS, and CAMS-R. Afterward, participants were randomly assigned to one of the three conditions: mindfulness, worrying, and mind-wandering. The worrying condition was included to mimic ruminative and catastrophic thinking that may result from distressing thoughts, while the mind-wandering condition acted as a neutral condition in which the participant idly distracts themselves from perseverating on a given thought for more than a minute or so at a time. In the mindfulness condition, participants underwent a brief mindfulness induction 
by following recorded instructions. The recorded instructions were taken from a guided meditation used by neuroscientist Sam Harris (2013). The meditation exercise directed the participant's attention to breathing and immediate bodily sensations, instructing the participant to simply maintain an awareness of their sensory experience rather than trying to control it (e.g., by controlling one's rate of inhalation/exhalation). This method is derived from Vipassana meditation and is designed specifically to cultivate mindful attention and awareness. In the worrying condition, participants listened to a 10-minute audio recording in which they were instructed to imagine their current worries or concerns across a variety of domains (relationships, personal achievement, health, and personal safety). Participants were instructed to imagine the outcome of the worst-case scenario that could possibly result from each of these concerns and consider the likelihood of each worry occurring in reality. This catastrophizing technique was adapted from methods used by Vasey and Borkovec (1992). In the mind-wandering condition, participants were instructed to allow their mind to wander aimlessly, with instructions to continue to allow their mind to wander repeated approximately once per minute. Each manipulation lasted approximately 10 minutes and recorded instructions for all manipulations were presented via headphones. After the experimental manipulation, all participants were asked to rate how closely they attempted to follow the instructions presented by each manipulation on a 7-point Likert-type scale.

After the randomly assigned experimental manipulation, all participants underwent a mortality salience induction using the procedure described by Niemiec et al. (2010). Participants were asked to "Briefly describe the emotions that the thought of your own death arouses in you... Jot down, specifically as you can, what you think will happen to you as you die and once you are dead." (Niemiec et al., 2010, p. 347). Next, participants completed the PANAS a second time, followed by the MSTS and the word fragment task to assess distal and proximal defense responses. Participants then completed the PANAS a third time and were given the opportunity to ask questions about the study before leaving. No noteworthy difficulties in completing any portion of the study were noted in any of the participants. The vast majority of participants completed all items in a prompt manner and relatively few participants posed any questions regarding the study when given the opportunity. A visual summary of the experimental procedure is depicted in Figure 1.

\section{Results}

\section{Statistical Analysis}

Analysis began with computation of correlations between trait mindfulness and the three dependent variables to assess for potential covariates to be used in the subsequent analyses. Trait mindfulness scales which correlated with a dependent 


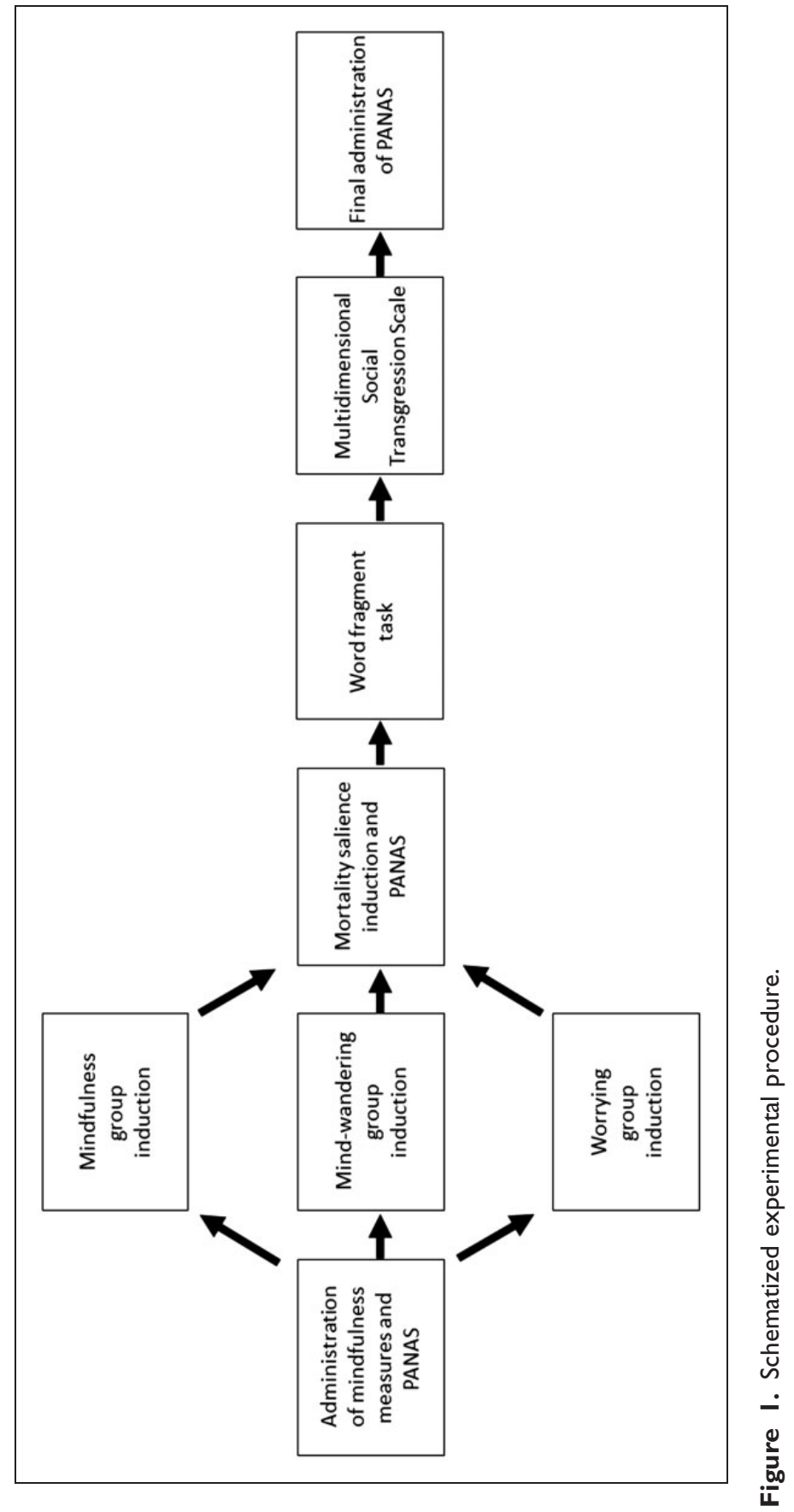


Table I. Intercorrelations for Three Trait Mindfulness Measures and Seven Dependent Variables.

\begin{tabular}{lccc}
\hline Measure & PHLMS & KIMS & CAMS-R \\
\hline I. Death-related words & -.139 & $-.348 * *$ & -.193 \\
2. Interpersonal offense severity & .173 & .215 & .139 \\
3. Interpersonal punishment severity & .087 & .160 & .009 \\
4. Intrapersonal offense severity & $.300^{*}$ & $.289 *$ & .229 \\
5. Intrapersonal punishment severity & .188 & .230 & .178 \\
6. Negative affect Time 2 & -.111 & -.040 & $-.35 I^{* *}$ \\
7. Negative affect Time 3 & -.101 & -.060 & $-.33 I^{* *}$ \\
\hline
\end{tabular}

Note. PHLMS = Philadelphia Mindfulness Scale; KIMS = Kentucky Inventory of Mindfulness Scales; CAMS$\mathrm{R}=$ Cognitive and Affective Mindfulness Scale-Revised.

$*_{p}<.05$. ** $p<.01$.

variable were entered as covariates in analyses utilizing that variable alone. A one-way multivariate analysis of covariance (MANCOVA) was used to assess for effects of experimental condition on negative affect, while a series of followup analysis of covariance's (ANCOVA) were used to examine each isolated time point identified as significant in the MANCOVA. An ANCOVA was used to assess for effects of condition on number of death-related words completed during the word fragment task. An analysis of variance was used to measure effects of condition on responses to the MSTS.

\section{Correlations of Trait Mindfulness With Dependent Variables}

Correlations were used to examine the relation between trait mindfulness, as measured by the three trait mindfulness scales and the three main dependent variables (negative affect at Time 1 and Time 2, number of death-related words written during the word fragment task, and MSTS score). These correlations are presented in Table 1.

As seen in Table 1, a significant negative relation was found between the CAMS-R total score and negative affect at Time 2 (immediately after the mortality salience induction), $r(75)=-.351, p=.002$, and Time 3 (at the end of the study), $r(73)=-.331, p=.004$. A significant negative relation was also found between the KIMS and number of death-related words written during the word fragment task, $r(62)=-.348, p=.005$. A positive relation was found between perceived severity of personal offenses and both the KIMS, $r(59)=.289$, $p=.024$, and the PHLMS, $r(68)=.300, p=.012$.

Because there was a significant negative relation between mindfulness (CAMS-R) and negative affect after the mortality salience induction as well as 
at the end of the experiment, CAMS-R scores were entered as a covariate in subsequent analyses when negative affect was the dependent variable.

\section{Baseline Negative Affect}

A one-way ANOVA was conducted to confirm that baseline negative affect did not differ across the three experimental conditions. No significant main effect was present, $F(2,73)=.787, p=.459$, indicating that all three experimental groups possessed equivalent baseline levels of negative affect.

\section{Participant Effort}

A one-way ANOVA was conducted to assess for differences across experimental conditions on participant ratings of how closely they attempted to follow the instructions presented in each experimental manipulation. No significant differences were found between the mindfulness, $(M=5.57, S D=1.16)$, mind-wandering $(M=6.07, S D=.874)$, and worrying $(M=5.69, S D=1.09)$ conditions, $F(2,73)=1.66, p=1.99$, indicating that all three groups were equally effortful in following the presented instructions during the experimental manipulation. This indicates that a moderately high level of effort was made by all three groups.

\section{Effect of Experimental Condition on Negative Affect}

A one-way MANCOVA was conducted to assess the effect of experimental condition (mindfulness, mind-wandering, and worrying) on PANAS negative affect scores at Times 2 and 3 with trait mindfulness (CAMS-R) entered as a covariate. A significant multivariate effect was observed, Wilks' $\lambda=.857, F(4$, $138)=2.679, p=.030$, partial $\eta^{2}=.074$. The results of the MANCOVA and follow-up ANCOVA's are presented in Table 2.

Given the significant multivariate effect, univariate ANCOVA's were used as a follow-up to the MANCOVA, with trait mindfulness (CAMS-R) entered as a covariate, examining the effect of experimental condition on negative affect at each of the three time points separately. A significant effect was found at Time 2, $F(2,69)=5.825, p=.005$, but not at Time 3, indicating significant differences in negative affect between at least two experimental conditions at Time 2 (directly after the mortality salience induction). Post hoc comparisons for the Time 2 analysis using Fisher's LSD indicated significantly less negative affect in the mindfulness condition than in the worrying condition at Time 2, with a mean difference of $-4.71, p=.003$ (see Figure 2), but no significant difference between the mindfulness and mind-wandering condition was found.

A follow-up repeated measures ANOVA was conducted to examine whether participants in the mindfulness condition experienced a decrease in negative affect over the course of the experiment. No significant change in negative 
Table 2. Means, Standard Deviations, and Analyses of Variance for the Effects of Mindfulness, Mind-Wandering, and Worrying Conditions on Nine Dependent Variables.

\begin{tabular}{|c|c|c|c|c|c|c|c|c|c|}
\hline \multirow[b]{2}{*}{ Variable } & \multicolumn{2}{|c|}{ Mindfulness } & \multicolumn{2}{|c|}{ Mind-Wandering } & \multicolumn{2}{|c|}{ Worrying } & \multirow[b]{2}{*}{$F$} & \multirow[b]{2}{*}{$p$} & \multirow{2}{*}{$\begin{array}{c}\text { Partia } \\
\eta^{2}\end{array}$} \\
\hline & $M$ & $S D$ & M & $S D$ & M & $S D$ & & & \\
\hline Negative affect Time I & 14.00 & 5.28 & 16.11 & 7.65 & 13.96 & 4.97 & .978 & .381 & .028 \\
\hline Negative affect Time 2 & 13.09 & 3.50 & 15.89 & 5.24 & 18.46 & 6.57 & 5.825 & .005 & .144 \\
\hline Negative affect Time 3 & 12.36 & 3.19 & 13.96 & 5.42 & 14.92 & 4.73 & 1.574 & .215 & .044 \\
\hline Number of death-related words & 2.26 & .991 & 2.26 & 1.287 & 2.95 & .921 & 4.613 & .014 & .135 \\
\hline Interpersonal transgression severity & 43.16 & 2.99 & 43.13 & 4.24 & 44.67 & 5.16 & .972 & .384 & .029 \\
\hline $\begin{array}{l}\text { Interpersonal transgression } \\
\text { punishment }\end{array}$ & 42.63 & 3.61 & 42.88 & 4.64 & 43.25 & 5.33 & .097 & .907 & .003 \\
\hline Intrapersonal transgression severity & 43.16 & 4.03 & 42.08 & 4.79 & 43.87 & 4.78 & .927 & .401 & .028 \\
\hline $\begin{array}{l}\text { Intrapersonal transgression } \\
\text { punishment }\end{array}$ & 40.26 & 4.92 & 40.83 & 5.09 & 41.25 & 5.43 & .193 & .825 & .006 \\
\hline
\end{tabular}

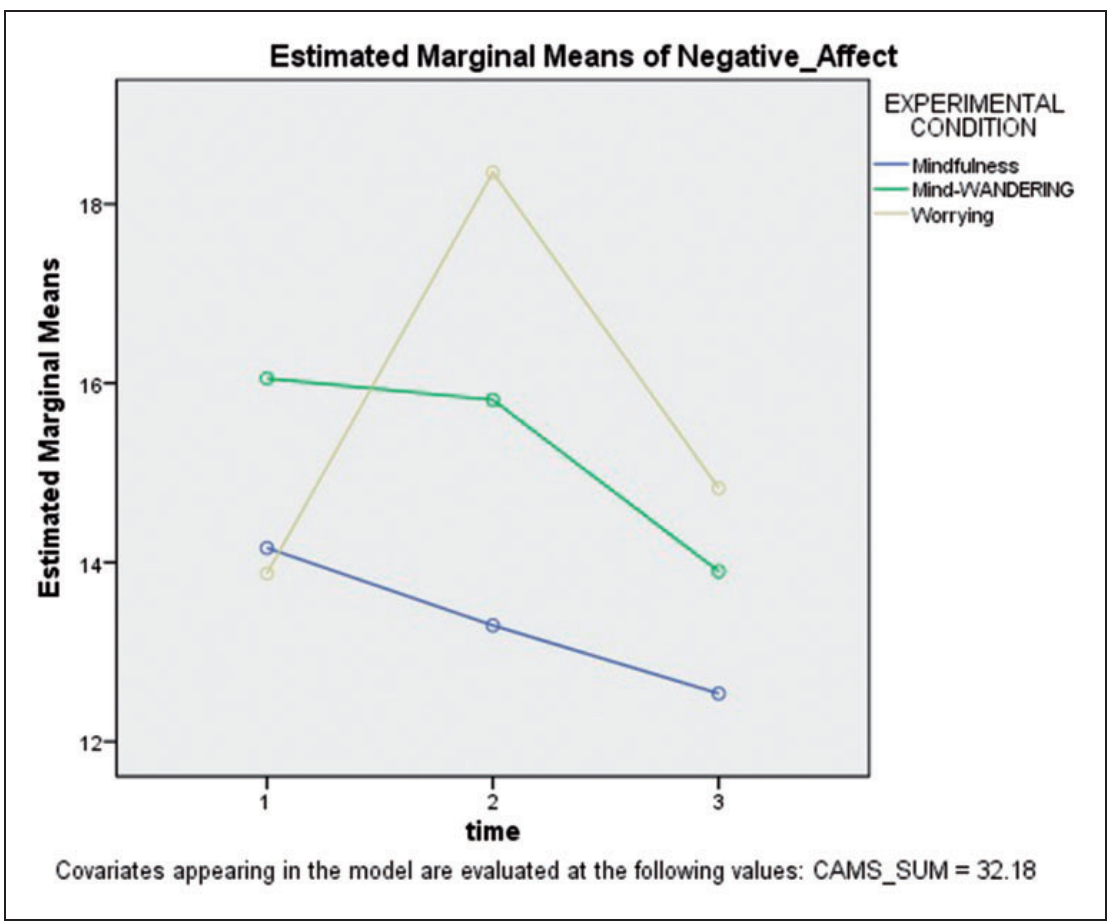

Figure 2. Negative affect across experimental conditions at Times I, 2, and 3. 
affect was found between any of the three time points for individuals in the mindfulness condition, $F(2,42)=2.26, p=.11$.

\section{Effect of Experimental Condition on Number of Death-Related Words Completed}

A follow-up ANCOVA was also conducted to examine the effect of experimental condition on number of death-related word fragments completed on the fragment task, with KIMS scores entered as a covariate. A significant effect was found, $F(2,59)=4.613, p=.014$. Post hoc comparisons using Fisher's LSD assessed specific differences between each of the three experimental conditions. Post hoc comparisons indicated fewer death-related words completed for the mindfulness condition $(M=2.26, S D=.99)$ compared with the worrying condition $(M=2.95, S D=.921)$, a mean difference of .78 that was statistically significant $(p=.017)$. Pairwise comparisons also indicated more death-related words completed in the mind-wandering condition $(M=2.26, S D=1.28)$ compared with the worrying condition, with a mean difference of .85 that was statistically significant $(p=.007)$. No significant difference in the number of completed word fragments was found between the mindfulness and mind-wandering conditions (see Figure 3).

\section{Effect of Experimental Condition on Response to Moral Transgression}

A follow-up ANOVA was conducted to examine the effect of experimental condition on participants' responses to the MSTS. Experimental condition was not found to have a significant effect on participants' responses when simultaneously separating scores across both severity and punishment as well as across interpersonal versus intrapersonal transgressions.

\section{Discussion}

The present study sought to investigate whether a brief mindfulness induction would buffer defensive responses to mortality salience. Specifically, defensive responses to mortality salience for those who underwent a mindfulness induction were compared with those who underwent either a mind-wandering or worry induction. Negative responses were measured in three ways: proximal defense mechanisms, as measured by a word fragment task in which a higher number of death-related words completed implied a more defensive response; distal defense mechanisms, in which higher ratings of offense severity and deserved punishment on the MSTS implied a more defensive response; and negative affect, as measured by the PANAS. Individuals in the worrying condition expressed more proximal defense responses than individuals in the mindfulness and mindwandering conditions who did not differ. Likewise, participants in the worrying 


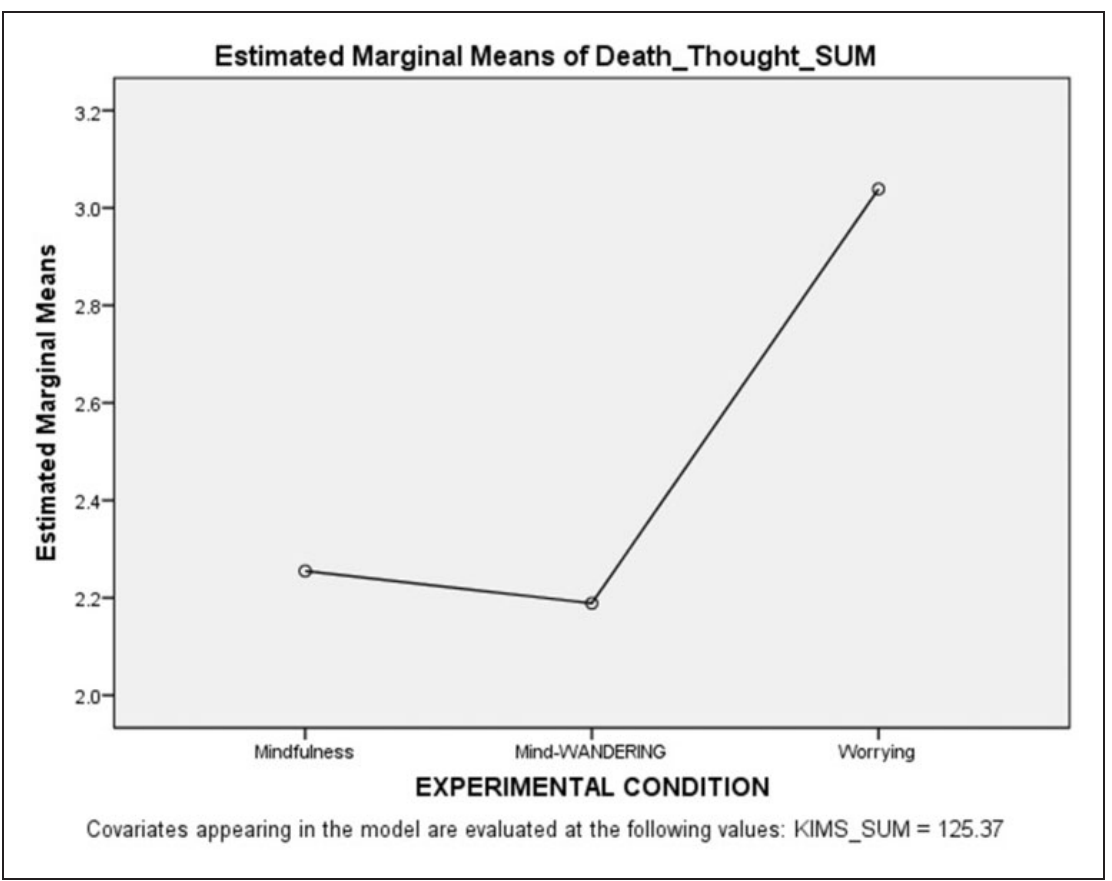

Figure 3. Mean completed word fragments across experimental conditions.

condition endorsed higher levels of negative affect after the mortality salience induction than did individuals in the mindfulness or mind-wandering condition who did not differ. No significant group differences were found for measures of distal defensive responses.

Partial support was found for the hypothesis that individuals in the mindfulness group would complete fewer death-related word fragments relative to other groups. Although participants in the mindfulness group completed significantly fewer death-related word fragments than those in the worrying group, there was no statistically significant difference between the mindfulness and mind-wandering groups. The most that can be concluded is that both the mindfulness and mind-wandering conditions were more effective than active worrying when confronted with mortality salience. Given that the mind-wandering condition was essentially neutral in terms of emotional manipulation, it is possible that this effect reflects an enhancement of death-related anxiety as a result of active worrying rather than an indication that either mind-wandering or mindfulness produced a buffering effect.

No support was found for the hypothesis that individuals in the mindfulness condition would endorse less severe punishments toward the hypothetical antagonists of the MSTS and would interpret the moral transgressions described 
in the MSTS as less severe in nature. No differences were found between any of the three groups when comparing their respective scores on this measure.

It is unclear as to why mindfulness was found to be more effective at reducing proximal defense responses and negative affect compared with worrying but had no significant effect on distal defense responses. However, some consideration must be given to the fact that studies utilizing the MSTS are sparse, and further studies probably should be conducted using the measure before to better determine if it is appropriate as a measure of distal defense responses to mortality salience. Furthermore, Niemiec et al. (2010) found that the MSTS only yielded significant differences in response patterns between a mortality salience condition and a control condition when comparing individuals with below average trait mindfulness. Accordingly, no difference in response patterns was found in individuals with high trait mindfulness. Thus, it may be worthwhile to consider the possibility that scores on the MSTS may be more sensitive to influence from trait mindfulness as a broader personality trait than to a brief experimental manipulation intended to increase state mindfulness. However, both the PHLMS and KIMS measures of trait mindfulness were significantly negatively correlated with intrapersonal offense severity as measured by the MSTS, and thus opposite direction than would be expected. Furthermore, Niemiec et al. (2010) found that MSTS scores only differed between individuals who had and had not been exposed to a mortality salience induction when such individuals scored low $(-1 S D)$ on trait mindfulness. Conversely, Niemiec et al. found no difference in MSTS scores in individuals with high trait mindfulness $(+1 S D)$ regardless of whether or not they had undergone a mortality salience induction. It is also worth reiterating that the MSTS was originally constructed for and tested with a sample of Israeli undergraduate students and that the number and content of questions used in the original Florian and Mikulincer's (1997) study differs from the measure as used by Niemiec et al. (2010) and in the present study, given that some items that were culturally irrelevant to the current study's sample were deleted. Thus, the form of the MSTS as used in the present study may not be functioning or have the same degree of validity as in previous studies.

Partial support was found for the hypothesis that under mortality salience, individuals in the mindfulness condition would report lower levels of negative affect relative to the other conditions. Mindfulness was found to be more effective than worrying in diminishing negative responses to mortality salience but was not significantly different from mind-wandering. With this in mind, an examination of the separation between negative affect scores across conditions at Time 2 does allow for the possibility of a noteworthy division between the three conditions that was not detected due to lack of statistical power (see Figure 2). It is hypothesized that, given a larger sample size, this pattern would hold and achieve statistical significance. Along the same line of thought, it is not out of the question to hypothesize that a larger sample size would yield 
significant results when assessing whether the mindfulness induction resulted in a steady decrease in negative affect from baseline to termination of the study. Based on a post hoc power analysis, and assuming the current effect sizes hold, approximately 81 participants would be needed in both the mindfulness and mind-wandering groups for the Time 2 negative affect differences across the groups to be statistically significant.

Nonetheless, the present study has yielded insufficient evidence to conclude that a brief mindfulness exercise is superior to simple distraction for buffering the negative defensive responses to mortality salience. Rather than saying that mindfulness produces a more positive effect than mind-wandering, the most that can be confidently said is that worrying produces a more negative effect than the other two alternatives. At least three potential explanations exist for this outcome: The first possibility is that a brief mindfulness exercise, when applied by an untrained individual, is simply insufficient at yielding a sufficient level of state mindfulness to buffer the negative effects more than for simply mind-wandering, which could be seen as a type of distraction.

Second, the motivation of the participants in the mindfulness condition must be taken into account. Some degree of concentrated attention is required to utilize mindfulness skills, whereas no effort or skill is necessary to engage fully in the mind-wandering condition. This is noteworthy given that the participants had little incentive to fully pay attention or follow the instructions in the study. With this in mind, most participants in the mindfulness condition reported a relatively high degree of effort in following the instructions presented in the mindfulness meditation exercise. A possible consideration is that, regardless of one's motivation, self-inducing a mindful state is a skill that requires sustained practice over time. A participant's effort in following the instructions presented may not reflect their actual success in doing so. It is therefore possible that a stronger effect would be seen in the mindfulness group after a sustained period of practice in utilizing mindfulness techniques. Thus, a future study comparing naive with experienced meditators could shed light on this issue.

\section{Limitations}

The present study must be considered within the context of at least five limitations. First, the study may have suffered from a small sample size relative to the size of the effect that was observed, resulting in a lack of statistical significance for an effect that may be deemed noteworthy. The present sample size was chosen based on a power analysis using a medium effect size, which is what was achieved when comparing the mindfulness group to the worry group, but not when comparing the mindfulness to the mind-wandering group. Approximately 20 to 27 individuals were included in each condition across all statistical analyses, with one isolated incident in which only 19 participants in the mindfulness condition could be included in the word fragment task analyses 
due to participant error in completing the task which rendered some data unusable. By way of contrast, recent studies by Niemiec et al. (2010) utilized sample sizes of sometimes over 200 participants, split up across 2 to 4 groups. These sample sizes occasionally surpassed the necessary number of participants to achieve significance given his achieved effect sizes - for example, Niemiec's study utilizing the MSTS, with a sample size of 128 , reported significant absolute value $\beta$ coefficients of between .18 and .28. Replications of the present study should strive to attain large sample sizes so as to increase statistical power.

Second, as stated before, the MSTS may not have been the optimal means of investigating distal defense responses to the mortality salience induction. Unlike the word fragment task, the MSTS has been utilized in comparatively few studies. A study by Niemiec et al. (2010) found no difference in MSTS scores between mortality salient participants and neutral participants when participants were high in trait mindfulness but did find a difference in score when participants were low in trait mindfulness. This indicates that MSTS score may be moderated by trait mindfulness. Accordingly, it may be that trait mindfulness has a greater effect on MSTS score than a brief mindfulness induction may be able to achieve. The series of studies which originally proposed use of the measure (Florian \& Mikulincer, 1997) was conducted using a larger set of items than the present study and utilized a sample of notably different culture, ethnicity, and nationality. Thus, the evidence garnered from the present study's analysis using the MSTS may be of limited use.

Third, it is worth noting that, because the PANAS was not administered between the experimental condition and the mortality salience induction, it is difficult to ascertain the extent to which negative affect was changed by the mortality salience induction itself versus the experimental condition inductions themselves. Nonetheless, differences across groups on the word fragment task do seem to indicate that the mortality salience induction had at least some lasting effect.

Fourth, it is noteworthy that the sample used in the present study consisted of predominately female participants. A total of 15 of the 77 participants were men, and after assigning each of the 15 men to one of the three experimental conditions, there were not enough men in the sample to reliably compare effects between men and women. Because of the sample's limited capacity to compare effects by gender, it cannot be said with high confidence that the present study is fully representative of both men and women.

Fifth, the sample used in the present study utilized convenience sampling to obtain participants. Because all participants were self-selected volunteers, it is possible that the present study was vulnerable to self-selection bias or other influences beyond the control of the researchers. Use of more advanced sampling techniques during data collection may mitigate these risks in future research. 


\section{Future Directions}

Although the present study provides noteworthy evidence suggesting the effectiveness of mindfulness over worry and perseveration when confronted with death-related anxiety, the results provide no evidence that mindfulness yields significant benefits when compared with simply distracting oneself from threatening thoughts. The results of the present study are currently unclear as to whether the lack of evidence supporting mindfulness's superiority over mindwandering, particularly in the realm of diminishing and guarding against negative affect, was due to a true lack of difference between the two conditions or whether a lack of statistical power could partially explain the lack of significant results. This question is particularly interesting in light of research indicating that mindfulness may be especially effective at reducing distracted and ruminative thinking, indicating that the mindfulness and mind-wandering conditions, while achieving similar results, may be doing so via differing pathways (Jain et al., 2007). Therefore, future studies should utilize a larger sample size to specifically investigate differences between mindfulness and neutral conditions in the context of death-related anxiety.

It is plausible that some of the anxiety-reducing benefits generated by the mindfulness induction were secondary to the relaxation response (Benson, 1975). Given the similarity between mindfulness meditation and relaxation training, it is recommended that future studies compare these two techniques directly. While the results of this comparison are as yet unclear, it is likely that one would see some notable improvement in negative affect among members of a "relaxation condition," given research indicating the efficacy of relaxation techniques in reducing anxiety (Luebbert, Dahme, \& Hasenburg, 2001; Wallace, Benson, \& Wilson, 1984).

One potentially fruitful question for future research is whether a stronger effect might be found if the mortality salience induction preceded the experimental manipulation. The effects of the mindfulness condition, presented immediately after the mortality salience condition, may more effectively counteract anxiety instilled by the mortality salience induction than in the present study. Conversely, the present study sought only to buffer the effects of mortality salience. A future study in which the order of the mortality salience induction and the experimental manipulation were reversed may compare the effect of utilizing mindfulness skills in response to distressing stimuli versus prior to distressing stimuli. Such an approach would examine the capacity for mindfulness to "undo" the negative effects of mortality salience, similar to the ability of positive emotions to undo the negative effects of negative emotions (Fredrickson, Mancuso, Branigan, \& Tugade, 2000)

A few noteworthy correlations between trait mindfulness and the other dependent variables were also found by the present study. In particular, trait mindfulness was highly negatively correlated with number of death-related 
words completed on the word fragment task, indicating that mindfulness as a personality trait may play a significant part in modifying internalized defense mechanisms in response to mortality salience. Likewise, trait mindfulness was highly negatively correlated with negative affect at both time points after the mortality salience induction, also indicating that mindfulness as a personality trait may similarly play a large part in buffering negative affective response to stimuli which invoke death-related anxiety. It is worth reiterating that these correlations were compared with the total sample, regardless of condition, indicating that trait mindfulness may be a larger predictor of response to mortality salience than individual approach to dealing with mortality salience (e.g., mindfulness, mind-wandering, and worrying) in certain contexts. Such findings appear commensurate with prior research which indicates that higher levels of mindfulness are associated with lower levels of anxiety (Chambers et al., 2009).

Research by Niemiec et al. (2010) found a significant interaction between trait mindfulness and experimental condition when measuring MSTS scores across participants who had undergone a mortality salience induction and those in a control group. Given the correlational significance of trait mindfulness as it pertains to the present study, the field would benefit from future studies examining the extent to which trait mindfulness may moderate the effect of the experimental manipulation.

As stated earlier, mindfulness skills must be diligently practiced over an extended period of time in order to build enough skill to exact maximum benefit. The present study utilized inexperienced undergraduate students with no previous training in mindfulness skills. It is possible that the mindfulness condition would yield larger effects if it utilized participants who practiced mindfulness skills regularly or had received formal training. A study in which inexperienced participants regularly practiced mindfulness skills may find more profound changes in response to mortality salience when comparing defensive responses and negative affect over multiple time points. Likewise, a study which exclusively utilized experienced meditators as participants may yield larger effects than the present study, which was limited to individuals with no prior experience or training in mindfulness skills.

Future research, including replications of the present study, should include a sample of men great enough to conduct reliable analysis of effects by gender as well as by experimental condition. Utilization of a sample with a significant male demographic will allow investigation into the extent to which the present results are representative of both men and women.

\section{Conclusions}

The present study sought to examine the effect of a brief mindfulness induction on responses to mortality salience. By administering a mortality salience induction after the mindfulness induction, participants' responses to mortality 
salience could be measured in the context of whether they had received a mindfulness induction or other experimental treatment (mind-wandering and worrying). Results indicate that mind-wandering and mindfulness are both superior to worry and perseveration in guarding against negative affect as well as in diminishing proximal defense mechanisms in response to mortality salience. Results were insufficient to conclude, however, that mindfulness is a superior approach to simple distraction when confronted with death-related anxiety. With that in mind, some preliminary data point toward possible future demonstration of the superior efficacy of mindfulness in dealing with these difficult thoughts, given enough statistical power. Correlations examined during the course of the study also indicate that trait mindfulness may be a significant predictor of individual responses to mortality salience, and future research should examine the extent to which trait mindfulness versus behavioral response to mortality salience affects final outcomes.

\section{Declaration of Conflicting Interests}

The author(s) declared no potential conflicts of interest with respect to the research, authorship, and/or publication of this article.

\section{Funding}

The author(s) received no financial support for the research, authorship, and/or publication of this article.

\section{References}

Arch, J. J., \& Craske, M. G. (2006). Mechanisms of mindfulness: Emotion regulation following a focused breathing induction. Behaviour Research and Therapy, 44(12), 1849-1858.

Arndt, J., Greenberg, J., Pyszczynski, T., \& Solomon, S. (1997). Subliminal exposure to death-related stimuli increases defense of the cultural worldview. Psychological Science, 8(5), 379-385.

Arndt, J., Schimel, J., \& Goldenberg, J. L. (2003). Death can be good for your health: Fitness intentions as a proximal and distal defense against mortality salience1. Journal of Applied Social Psychology, 33(8), 1726-1746.

Baer, R. A., Smith, G. T., \& Allen, K. B. (2004). Assessment of mindfulness by self-report the Kentucky inventory of mindfulness skills. Assessment, 11(3), 191-206.

Benson, H. (1975). The relaxation response. New York, NY: Morrow.

Brown, K. W., Ryan, R. M., \& Creswell, J. D. (2007). Mindfulness: Theoretical foundations and evidence for its salutary effects. Psychological Inquiry, 18(4), 211-237.

Burke, B. L., Martens, A., \& Faucher, E. H. (2010). Two decades of terror management theory: A meta-analysis of mortality salience research. Personality and Social Psychology Review, 14(2), 155-195.

Cardaciotto, L., Herbert, J. D., Forman, E. M., Moitra, E., \& Farrow, V. (2008). The assessment of present-moment awareness and acceptance: The Philadelphia mindfulness scale. Assessment, 15(2), 204-223. 
Chambers, R., Gullone, E., \& Allen, N. B. (2009). Mindful emotion regulation: An integrative review. Clinical psychology review, 29(6), 560-572.

Coolsen, M. K., \& Nelson, L. J. (2002). Desiring and avoiding close romantic attachment in response to mortality salience. Journal of Death and Dying, 44(3), 257-276.

Feldman, G., Hayes, A., Kumar, S., Greeson, J., \& Laurenceau, J. P. (2007). Mindfulness and emotion regulation: The development and initial validation of the Cognitive and Affective Mindfulness Scale-Revised (CAMS-R). Journal of Psychopathology and Behavioral Assessment, 29(3), 177-190.

Florian, V., \& Mikulincer, M. (1997). Fear of death and the judgment of social transgressions: A multidimensional test of terror management theory. Journal of Personality and Social Psychology, 73(2), 369.

Fredrickson, B. L., Mancuso, R. A., Branigan, C., \& Tugade, M. M. (2000). The undoing effect of positive emotions. Motivation and Emotion, 24, 237-258.

Gailliot, M. T., Schmeichel, B. J., \& Baumeister, R. F. (2006). Self-regulatory processes defend against the threat of death: Effects of self-control depletion and trait selfcontrol on thoughts and fears of dying. Journal of Personality and Social Psychology, 91(1), 49.

Greenberg, J., Pyszczynski, T., \& Solomon, S. (1986). The causes and consequences of a need for self-esteem: A terror management theory. In R. F. Baumeister (Ed.), Public self and private self (pp. 189-212). New York: Springer.

Greenberg, J., Pyszczynski, T., Solomon, S., Simon, L., \& Breus, M. (1994). Role of consciousness and accessibility of death-related thoughts in mortality salience effects. Journal of Personality and Social Psychology, 67(4), 627.

Greenberg, J., Simon, L., Pyszczynski, T., Solomon, S., \& Chatel, D. (1992). Terror management and tolerance: Does mortality salience always intensify negative reactions to others who threaten one's worldview? Journal of Personality and Social Psychology, 63(2), 212.

Greenberg, J., Solomon, S., \& Pyszczynski, T. (1997). Terror management theory of selfesteem and cultural worldviews: Empirical assessments and conceptual refinements. Advances in Experimental Social Psychology, 29, 61-139.

Grossman, P., Niemann, L., Schmidt, S., \& Walach, H. (2004). Mindfulness-based stress reduction and health benefits: A meta-analysis. Journal of Psychosomatic Research, 57(1), 35-43.

Harris, S. (2013). Guided meditation with Sam Harris-Short version [Audio]. Retrieved from https://www.youtube.com/watch?v=pzMhLmErz5Q

Jain, S., Shapiro, S. L., Swanick, S., Roesch, S. C., Mills, P. J., Bell, I., \& Schwartz, G. E. (2007). A randomized controlled trial of mindfulness meditation versus relaxation training: Effects on distress, positive states of mind, rumination, and distraction. Annals of Behavioral Medicine, 33(1), 11-21.

Kabat-Zinn, J. (1994). Wherever you go, there you are: Mindfulness meditation in everyday life. New York, NY: Hyperion Books.

Kashdan, T. B., \& Rottenberg, J. (2010). Psychological flexibility as a fundamental aspect of health. Clinical Psychology Review, 30(7), 865-878.

Kesebir, P. (2014). A quiet ego quiets death anxiety: Humility as an existential anxiety buffer. Journal of Personality and Social Psychology, 106(4), 610. 
Luebbert, K., Dahme, B., \& Hasenbring, M. (2001). The effectiveness of relaxation training in reducing treatment-related symptoms and improving emotional adjustment in acute non-surgical cancer treatment: A meta-analytical review. Psycho-Oncology, 10(6), 490-502.

Niemiec, C. P., Brown, K. W., Kashdan, T. B., Cozzolino, P. J., Breen, W. E., LevesqueBristol, C., \& Ryan, R. M. (2010). Being present in the face of existential threat: The role of trait mindfulness in reducing defensive responses to mortality salience. Journal of Personality and Social Psychology, 99(2), 344.

Quaglia, J. T., Brown, K. W., Lindsay, E. K., Creswell, J. D., \& Goodman, R. J. (2015). From conceptualization to operationalization of mindfulness. In K. W. Brown, J. D. Creswell \& R. M. Ryan (Eds.), Handbook of mindfulness: Theory, research, and practice (pp. 151-170). New York, NY: Guilford Press.

Routledge, C., Arndt, J., \& Goldenberg, J. L. (2004). A time to tan: Proximal and distal effects of mortality salience on sun exposure intentions. Personality and Social Psychology Bulletin, 30(10), 1347-1358.

Sliter, M. T., Sinclair, R. R., Yuan, Z., \& Mohr, C. D. (2014). Don't fear the reaper: Trait death anxiety, mortality salience, and occupational health. Journal of Applied Psychology, 99(4), 759.

Vasey, M. W., \& Borkovec, T. D. (1992). A catastrophizing assessment of worrisome thoughts. Cognitive Therapy and Research, 16(5), 505-520.

Wallace, R. K., Benson, H., \& Wilson, A. F. (1984). A wakeful hypometabolic physiologic state. In D. H. Shapiro Jr. \& R. N. Walsh. (Eds.), Meditation: Classic and contemporary perspectives (pp. 417-431). New York, NY: Aldine.

Watson, D., Clark, L. A., \& Tellegen, A. (1988). Development and validation of brief measures of positive and negative affect: The PANAS scales. Journal of Personality and Social Psychology, 54(6), 1063.

\section{Author Biographies}

David M. Schultz is a PhD student in clinical psychology at The University of Southern Mississippi. His research interests include mindfulness, clinical personality assessment, and positive psychology.

Randolph C. Arnau is a professor of psychology at The University of Southern Mississippi. His research interests include positive psychology and clinical personality assessment. 